Costos operacionales de la ampliación del tamizaje prenatal de sífilis mediante sistemas rápidos en Bolivia y Mozambique

La sífilis causa graves trastornos durante el embarazo y es un importante cofactor en la transmisión del virus de inmunodeficiencia adquirida (VIH). El diagnóstico de la sífilis materna se basa en el tamizaje serológico de las mujeres embarazadas y su tratamiento consiste en una inyección única de penicilina. Si no se trata adecuada y oportunamente, esta infección puede provocar la muerte del feto, el parto prematuro o la infección congénita e incluso la muerte del recién nacido. En Bolivia, todas las mujeres tienen acceso al seguro universal materno-infantil, sin embargo, la mayoría de las clínicas rurales no ofrecen este servicio por carecer de la infraestructura de laboratorio necesaria.

Los sistemas rápidos de diagnóstico de sífilis basados en tiras inmunocromatográficas (TIC) ofrecen la posibilidad de fortalecer y ampliar el tamizaje prenatal de esta infección, especialmente en las zonas rurales, donde no hay condiciones adecuadas para realizar la prueba con reagina plasmática rápida (RPR). Para ello es necesario recabar antes información acerca de la efectividad en función del costo que pudiera tener la introducción del tamizaje de mujeres embarazadas mediante pruebas de sífilis basadas en TIC.

En este trabajo se compararon los costos de las pruebas con TIC y RPR en hospitales maternos urbanos con laboratorios de Bolivia y en clínicas de cuidados prenatales de Mozambique. Además, se evaluaron los costos de introducir la realización de las pruebas rápidas con TIC durante las visitas prenatales a los centros de salud rurales de ambos países que no cuentan con infraestructura de laboratorio o personal calificado para realizar la prueba con RPR.

Los datos de los costos se tomaron durante cuatro visitas de trabajo realizadas a los hospitales y clínicas participantes durante los años 2003 y 2004. La información acerca del número de mujeres tamizadas y tratadas en cada unidad durante la ejecución del proyecto se utilizó para calcular el incremento en los costos, según los datos y costos específicos de cada unidad. Tanto en Bolivia como en Mozambique se realiza el tamizaje y el tratamiento para la sífilis como parte de los servicios prenatales de salud, por lo que en este estudio se estimó solamente el incremento en el costo del tamizaje y el tratamiento de las mujeres que resultarían positivas a la prueba rápida con TIC.

De los 11618 tamizajes realizados durante el período de ejecución del proyecto en Bolivia, 8900 se realizaron en hospitales urbanos de maternidad. De ellas se trataron con penicilina $417(4,7 \%)$ que resultaron positivas según la prueba con TIC. En las instalaciones rurales sin laboratorio se tamizaron 2178 mujeres, de las cuales $121(5,6 \%)$ recibieron tratamiento contra la sífilis. Al realizar la confirmación de la infección, se encontró $23,0 \%$ de falsos positivos cuando se emplearon los resultados de la prueba con RPR y 29,0\% cuando se utilizaron los de la prueba con TIC.

El costo promedio del tamizaje en Bolivia fue de US $\$ 1,48$ con la prueba de RPR y de US $\$ 1,91$ con la de TIC. En los centros de salud sin laboratorio, el costo del tamizaje con la prueba de TIC fue de US $\$ 2,84$. No obstante, estos valores podrían disminuir considerablemente si se emplearan sistemas de diagnóstico más económicos.

El incremento en el costo por la ampliación del tamizaje a las mujeres embarazadas de zonas rurales en Bolivia —donde ya el gobierno apoya este programa con aproximadamente US $\$ 1,00$ por mujer estudiada - sería cuando más de US\$ 1,85 por cada mujer tamizada.

Según estos resultados, la ampliación del tamizaje para la infección de sífilis en las mujeres embarazadas de Bolivia mediante la introducción de sistemas rápidos basados en TIC en instalaciones que no cuentan con infraestructura de laboratorio es factible. El costo de la prueba con TIC es similar al de la prueba con PRP. La información brindada en este estudio es de gran importancia para los profesionales y funcionarios encargados de elaborar las políticas de salud pública en países y zonas que no disponen de recursos financieros y humanos suficientes para universalizar el tamizaje de sífilis en mujeres embarazadas. (Levin CE, Steele M, Atherly D, García SG, Tinajeros F, Revollo R, et al. Analysis of the operational costs of using rapid syphilis tests for the detection of maternal syphilis in Bolivia and Mozambique. Sex Transm Dis. 2007; 34(7):S47-54.)

\section{¿Pueden los cuidados intensivos reducir el índice de utilización hospitalaria de los pacientes con trastornos mentales graves?}

Los servicios modernos de salud mental se esfuerzan por garantizar que las personas con enfermedades mentales graves permanezcan hospitalizadas el menor tiempo posible, tanto para evitar gastos hospitalarios innecesarios como por la estigmatización que representa y el disgusto de los pacientes. Para lograr esto, los servicios de salud mental recurren cada vez más al uso de cuidados 